\title{
Bridging the Gap for Homebound Elderly Diabetics: Increasing Awareness of Interventions for Diabetic Homebound Elderly Adults
}

Received: Ocotber 01, 2015; Accepted: Ocotber 05, 2015; Published: Ocotber 12, 2015

According to the National Diabetes Statistics Report of 2014 [1], 21 million people in the United States are diagnosed with diabetes. Of this number, 11.2 million are 65 or older. This is of concern because the numbers of people age 65 years and over has increased by $24.7 \%$ from 35.9 million in 2003 to 44.7 million in 2013 [2], which leads to an increase in the average life span of persons reaching age 65 to 19.3 years. Despite this significant increase in life expectancy, diabetes significantly reduces the lifespan for men and women over 50 years by an average of 7.9 years. Nationally, the highest prevalence of diabetes is among the American Indians/Alaska Natives (15.9\%), followed by NonHispanic Blacks (13.2\%), and Hispanics (12.8\%). According to the Centers for Disease Control and Prevention (CDC) Report [3], diabetes is the largest and fastest-growing chronic disease in the nation and was the seventh leading cause of death by disease in the United States in 2007. In addition to diabetes, there is increased risk of suboptimal nutrition, hospitalizations, nursing home admissions, and physical disability among the elderly population [4]. It is estimated that $37-40 \%$ of individuals over age 65 years do not get adequate nutrients [5]. Some measures such as Meals-on-Wheels (MOW) are already in place to assist the elderly with their nutritional needs. Currently, the Older Americans Act (OAA) federally mandates MOW to supply only a minimum of $1 / 3$ of nutrients set by the Dietary Reference Intakes (DRI) and meet state and local food safety and sanitation requirements for individuals 60 years and older. Furthermore, this act fund $30 \%$ of the cost of home delivered meals [6]. Provision of specific therapeutic diets is not included in the DRI for any population groups, therefore the homebound elderly diabetic clients who receive their meals from MOW need additional help in managing their food intakes in relation to their health condition.

As stated by The National Home and Hospice Care Survey of 2011, about 7.6 million people receive home-based care and of those individuals, $30.6 \%$ are diagnosed with diabetes [7]. An estimated $7 \%$ of US adults over the age of 60 receive homedelivered meals through programs such as, Meals-on-Wheels and of those participants, $74 \%$ are at nutritional risk [8]. In 2014, MOW provided 61,045 meals to residents in DeKalb County, Illinois and surrounding areas. Despite efforts to provide meals to the homebound elderly population, those with diagnosed

\section{Sheila Barrett, Laura Lawler BS and Ashley Kyle AS}

School of Family, Consumer, and Nutrition Sciences, Northern Illinois University, DeKalb, IL, USA

Corresponding author: Sheila Barrett

School of Family, Consumer, and Nutrition Sciences, Northern Illinois University, DeKalb, Illinois, 60115, USA.

\section{sbarrett1@niu.edu}

Tel: $815-752-7063$

Fax: 815-753-1321

Citation: Barrett S, Laura Lawler BS, Ashley Kyle AS. Bridging the Gap for Homebound Elderly Diabetics: Increasing Awareness of Interventions for Diabetic Homebound Elderly Adults. J Clin Nutr Diet. 2016, 1:1

type 2 diabetes (T2D) require further assistance with managing their diabetes. In 2012, DeKalb County, Illinois had 6,106 cases of diagnosed diabetes. Research is needed on this population to determine the prevalence of diabetes and how homebound elderly diabetics are managing their condition. Homebound elderly residents include "community dwelling residents over 60 years of age and who do not live in Nursing Homes or Assisted Living Facilities."

There is no known cure for diabetes but it can be treated to enable diabetics to live better lives. Managing diabetes is necessary due to the high medical costs it incurs. The average medical expenses are more than twice as high for a person with diabetes when compared to those without diabetes because treatment usually requires patient education, special equipment and supplies. It can become costly especially when it is not covered by health insurance. In 2007, the estimated cost of treating diabetes in the United States was $\$ 174$ billion; $\$ 116$ billion in direct medical care costs and $\$ 58$ billion in indirect costs; from disability, productivity loss, and premature death [9]. However, early screening can lead to preventive measures, and lessen the costs associated with emergency room visits, hospitalizations, loss of sight, and loss of limbs. More importantly, diabetes is accompanied by other conditions such as hypertension, high total cholesterol (TC), high 
Low Density Lipoprotein Cholesterol (LDL-C), high triglycerides (TG), low High Density Lipoprotein Cholesterol (HDL-C), and overweight. Low physical activity also exacerbates the diabetes condition. Interventions are needed to educate patients and increase physical activity among this group.

Diabetes is a major cause of heart disease and stroke. Death rates for heart disease and the risk of stroke are about 2-4 times higher among adults with diabetes than among those without diabetes. In addition, $67 \%$ of U.S. adults who report having diabetes also report having high blood pressure [9]. For people with diabetes, high blood pressure, high cholesterol levels, and smoking increase the risk of heart disease and stroke [10]. Controlling blood pressure and cholesterol levels and smoking cessation can help to reduce this risk. More serious complications of diabetes have been related to vision loss, kidney failure, and amputations of legs or feet. Effective glucose control, as measured by $\mathrm{A} 1 \mathrm{c}$ levels, and blood pressure control can prevent or delay these complications. Additionally, preventive measures can reduce disease progression and ease the economic burden of diabetes treatment while improving the quality of life for all people who have, or are at risk of diabetes which are defined goals of Healthy People 2020.

Medicare, a federal program, covers some health care costs for individuals over age 65 or who have disabilities. It also covers diabetes self-management training and nutrition counseling services, lab testing, diabetes glucose testing supplies, and insulin pumps and associated supplies [11]. Medicare Part B covers diabetes outpatient self-management training to cope with, and manage diabetes. Training, covered up to ten hours after diagnosis, includes tips for eating healthy, increased physical activity, monitoring blood sugar, and taking medication. However, ten hours of training post diagnosis is not enough to effectively manage diabetes in the ensuing years, especially among homebound elderly residents. Along with the initial ten hours of training that is covered under Medicare, some individuals may qualify for an additional two hours of training per year if they meet specific criteria; if the training is provided to 2-20 persons at the same time, recommended by one's doctor, lasts for at least 30 minutes, and must occur after the calendar year in which one was diagnosed. Even though these two additional hours are available, due to these stipulations for coverage under Medicare Part B, many homebound elderly diabetics may not meet these criteria. For example, they may experience problems with mobility and are unable to attend and participate in training programs wherein 2-20 persons are in attendance.
It is important to facilitate the needs of homebound elderly diabetics so they can better manage their condition. More research is needed on how to reach this population group, while bearing in mind the peculiar characteristics of this group; lack of mobility, low income, lack of family and social support. One way of identifying homebound elderly diabetics is through collaboration with local programs such as MOW, which provides meals to people over 65 years of age. Once these individuals are identified, training programs can be put in place wherein the elderly can be transported to one common area; community centers or church fellowship halls. In DeKalb County, IL transportation can be economically provided by MEDVac. Similar modes of transportation may be available in other states. Furthermore, training materials can be obtained from the National Diabetes Education Program (NDEP), which is jointly sponsored by CDC and the National Institute of Health (NIH). A variety of educational tool kits and multimedia resources for managing and monitoring diabetes are available free of cost to health care professionals and diabetes educators. Researchers can optimize the use of these educational materials at no additional costs, thereby reducing financial strains on these homebound elderly residents. Instead of offering two hours of training per year, training sessions may be offered once per month for at least 30 minutes per session. Funding may be obtained from State-Based Diabetes Prevention and Control Programs supported by the CDC, which provides financial assistance and support to states for preventing, detecting and treating diabetes and its related complications. States may apply to CDC annually for available State Diabetes Prevention and Control Programs (DPCP) funds. At these training sessions, monitoring of high total cholesterol (TC), high Low Density Lipoprotein Cholesterol (LDL-C), high triglycerides (TG), low High Density Lipoprotein Cholesterol (HDL-C), and fasting blood glucose (FBG), may be achieved through testing with handheld monitoring devices which are reliable and cost effective, as long as funding is available to purchase these monitors.

In summary, with the increase in individuals living over 65 years of age, the growing obesity epidemic leading to T2D in younger populations may transcend into adulthood, which will lead to further complications regarding cost and managing this condition. Obesity-related medical conditions such as strokes, hypertension, and heart diseases exacerbate the problems of managing T2D. The economic burden of managing T2D warrants further intervention research. The homebound elderly diabetics need special attention in order to provide services that are accessible and affordable to them. 


\section{References}

1 National Diabetes Statistics Report, CDC. 2014; 1-12.

2 US Dept. of Health and Human Services, AoA Administration on Aging

3 Centers for Disease Control and Prevention. Diabetes Report Card 2012. Atlanta, GA: Centers for Disease Control and Prevention, US Department of Health and Human Services; 2012.

4 Rizvi A, 2009 Nutritional challenges in the elderly with diabetes. Int J of Diabetes Mellitus. 1:26-31.

5 Institute of Medicine, Committee on Nutrition Services for Medicare Beneficiaries. (199) The role of nutrition in maintaining health in the nation's elderly: Evaluating coverage of nutrition services for the Medicare population. Washington, D.C. National Academy Press.

6 Wellman NS, Rosenzweig LY, Lloyd JL, (2002) Thirty Years of Older Americans Nutrition Program. J Am Diet Assoc. 3:348-350.
7 Caffrey C, Sengupta M, Moss A, Harris-Kojetin L, Valverde R, (2011) Home Health Care and Discharged Hospice Care Patients: United States, 2000 and 2007. National Health Statistics Report CDC 38.

8 Moran MB, (2004) Challenges in the meals on wheels program. J Am Diet Assoc, 104:1219-1221.

9 Centers for Disease Control and Prevention, (2011) National Diabetes Fact Sheet. Atlanta, GA: Centers for Disease Control and Prevention, US Department of Health and Human Services

10 Imperatore G, Cadwell BL, Geiss LS, Saaddine JB, Williams DE, Ford ES, Thompson TJ, Narayan KM, Gregg EW et al. (2004) Thirty-year trends in cardiovascular risk factor levels among U.S. adults with diabetes. Am J Epidemiol. 160:531-539.

11 United States Census Bureau. Health Insurance Coverage Status by State for All People: 1999 to 2007. 